\title{
A Comparison of MDMA and Amphetamine in the Drug Discrimination Paradigm
}

\author{
D.N. Harper* , A. Crowther and S. Schenk \\ School of Psychology, Victoria University of Wellington, New Zealand
}

Keywords: MDMA, amphetamine, drug discrimination, rats.

\section{INTRODUCTION}

This study used the Drug Discrimination paradigm to assess the relative subjective experience of acutely administered 3,4-Methylenedioxymethamphetamine (MDMA) and d-amphetamine (AMPH). In this paradigm rats are required to respond on one lever if they have been given a specific drug (AMPH for one group of rats or MDMA for another group) $v s$ an alternate lever if they have been administered saline prior to a session. Generalisation and substitution testing was used to establish the extent to which rats previously trained to discriminate saline vs MDMA responded to novel exposure of AMPH. Likewise, we examined the extent to which rats previously trained to discriminate saline vs AMPH responded to novel exposure to MDMA. Previous research $[1,2]$ has demonstrated that rats can learn to discriminate MDMA from amphetamine, thus it was expected that only partial, if any, generalisation would be observed when exposed to an untrained novel drug. In addition, we examined the effect of concurrent administration of the D1 antagonist SCH23390 had on discrimination performance in both groups.

\section{METHODS}

\section{Subjects}

16 Sprague Dawley rats. 8 trained to discriminate between AMPH and saline, 8 trained to discriminate between MDMA \& saline.

\section{Training - Part 1}

Animals were administered a training dose of either AMPH $(0.5 \mathrm{mg} / \mathrm{kg}$ or $0 \mathrm{mg} / \mathrm{kg}, 15$ minute pre-treatment) or MDMA $(1.5 \mathrm{mg} / \mathrm{kg}$ or $0 \mathrm{mg} / \mathrm{kg}, 20$ minute pre-treatment) and placed in an operant chamber. Two retractable levers were inserted into the chamber. Levers were assigned as either drug or vehicle appropriate. Responding on the appropriate lever resulted in availability of a reinforcer on an FR10 schedule. Responding on the unpaired lever reset the FR for the correct lever. Generalisation \& Substitution testing started once animals were $80 \%$ correct prior to receiving the first reinforcer for 3 consecutive days.

*Address correspondence to this author at the School of Psychology, Victoria University of Wellington, New Zealand; Tel: +64 44635561; Fax: +64 44635402; E-mail: david.harper@vuw.ac.nz

\section{Generalisation \& Substitution Testing - Part 2}

Across a number of sessions all animals were administered a range of doses of $\operatorname{MDMA}(0,0.75,0.375$, or $1.5 \mathrm{mg} / \mathrm{kg})$ or AMPH $(0,0.375,0.25$, or $0.5 \mathrm{mg} / \mathrm{kg})$. Animals received each testing dose only once. Sessions ended at completion of the first 10 consecutive responses on either lever. (no reinforcers delivered).

\section{Discrimination Performance Following Concurrent Administration of D2 Antagonist - Part 3}

The D1 antagonist SCH23390 (0 - $0.1 \mathrm{mg} / \mathrm{kg})$ was administered concurrently with the appropriate training dose of AMPH or MDMA.

\section{RESULTS}

Animals trained to discriminate AMPH (left graph, Fig. 1) displayed full substitution at the training dose, partial substitution for lower doses, and no substitution for vehicle $(n=7)$. Partial substitution was also observed for the higher doses of MDMA, reducing in a dose-dependent manner $(n=5)$. Animals trained to discriminate MDMA (right graph) showed a similar dose dependent function of drug appropriate responding for MDMA as the AMPH group did for their training drug $(n=7)$, however, no dose of AMPH substituted for MDMA $(n=2)$. The D1 antagonist (SCH23390) reduced the ability of AMPH trained rats to discriminate AMPH from saline at doses that had no impact on the ability of MDMA trained rats to discriminate the training dose of MDMA from saline (although at the highest dose MDMA discrimination was also impaired).

\section{DISCUSSION \& CONCLUSION}

Higher doses of MDMA partially substituted for AMPH, however, no dose of AMPH substituted for MDMA. This suggests that MDMA induces some of the primary subjective experiences elicited by AMPH, but that the primary subjective experience of MDMA is markedly different from that of AMPH. The partial substitution on MDMA for $\mathrm{AMPH}$, as well as the relatively greater impact of the D1 antagonist on the AMPH trained group, suggests that the DA-agonist properties of both drugs may underlie this partial overlap in the subjective experience of both drugs. 


\section{Rats trained on AMPHETAMINE}

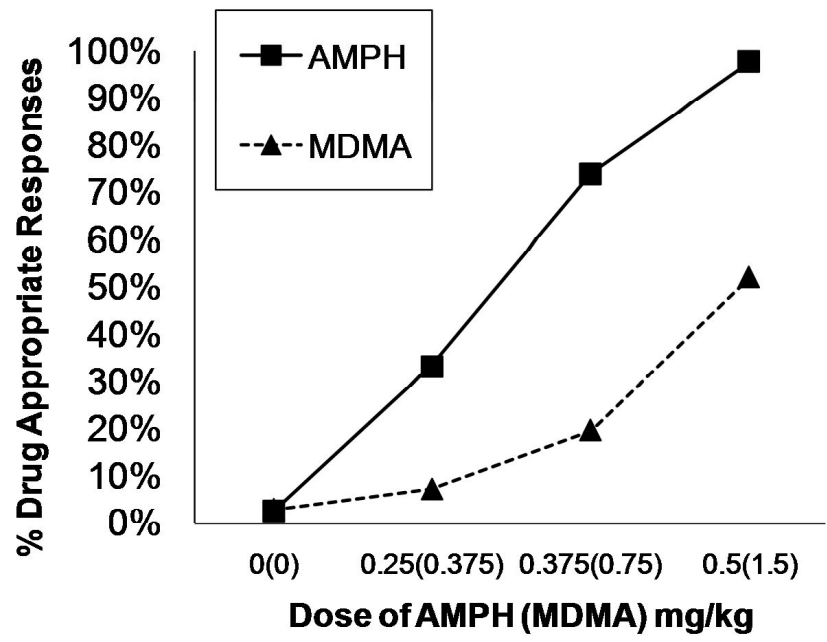

Rats trained on MDMA

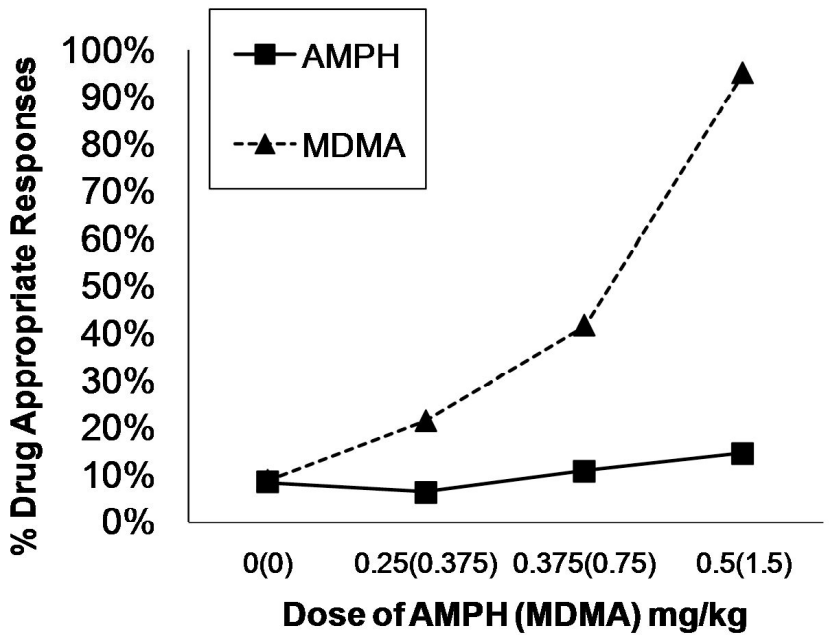

Fig. (1). Drug discrimination performance in rats trained to discriminate amphetamine from saline (left) or MDMA from saline (right).

\section{REFERENCES}

[1] Goodwin A, Baker L. A three-choice discrimination procedure dissociates the discriminative stimulus effects of d-amphetamine and ( \pm )-MDMA in rats. Exp Clin Psychopharmacol 2000; 8: 41523 .
[2] Goodwin A, Pynnonen D, Baker L. Serotonergic-Dopaminergic mediation of MDMA's discriminative stimulus effects in a threechoice discrimination. Pharmacol Biochem Behav 2003; 74: 98795.

(C) Harper et al.; Licensee Bentham Open.

This is an open access article licensed under the terms of the Creative Commons Attribution Non-Commercial License (http://creativecommons.org/licenses/ by-nc/3.0/) which permits unrestricted, non-commercial use, distribution and reproduction in any medium, provided the work is properly cited. 